\title{
Charge on the quantum dot in the presence of tunneling current
}

\author{
Mariusz Krawiec and Karol I. Wysokiński \\ Institute of Physics, M. Curie-Skłodowska University, \\ PL-20031 Lublin, Poland
}

November 3, 2018

\begin{abstract}
The calculation of the charge present in central region of the double barrier structure at non-equilibrium conditions is discussed. We propose here a simple method to calculate non equilibrium Green's functions which allows consistent calculations of retarded and distribution functions. To illustrate the approach we calculate the charge on the quantum dot coupled via tunnel barriers to two external leads having different chemical potentials $\mu_{L}$ and $\mu_{R}$. The obtained results have been compared with other approaches existing in the literature. They all agree in the equilibrium situation and the departures grow with increasing the difference $\mu_{L}-\mu_{R}$.

Keywords: A. nanostructures; D. electron - electron interactions; D. tunneling
\end{abstract}


Recent advancements of nanotechnology allow the design and study of devices in which quantum phenomena play primary role [1]. Typical device consists of a small central region coupled via tunnel barriers to two external electrodes (leads). In such quantum-dot devices Coulomb blockade, resonant tunneling [2] and Kondo effects [3, 4] have been observed. Theoretical description of electron transport phenomena in quantum dot often requires (self-consistent) calculations of the charge accumulated in the central region [5, 6]. If the system is in an equilibrium state (i.e. the chemical potentials of the left and right leads are the same $\mu_{L}=\mu_{R}=\mu$ ) the calculation of charge on the dot is easy. One finds the (local) density of states for the dot $N_{d}(\varepsilon)$ and integrates it with appropriate distribution function, which in equilibrium coincides with Fermi-Dirac one; $f(\varepsilon)=\left(e^{\beta(\varepsilon-\mu)}+1\right)^{-1}$, with $\beta=1 / k_{B} T$ and gets

$$
n=\int_{-\infty}^{\infty} d \varepsilon N(\varepsilon) f(\varepsilon) .
$$

The easiest way to derive (11) is to find the (equilibrium) Green's function [7] of the dot and apply spectral theorem to get $n$. In non equilibrium situation $\left(\mu_{L} \neq \mu_{R}\right)$ one can not use the equilibrium technique. Physically the problem is much more complicated as the tunneling current flows across the system and there is no a priori simple prescription as how to calculate the charge on the central region of the device (dot). Some additional complications arise if time dependent voltage or other time dependent fields are applied to the system. Here, however, we shall consider non-equilibrium but steady state. At nonequilibrium one can think about at least two ways of generalizing Eq. (1). The first is to replace the chemical potential $\mu$ by suitably chosen average chemical potential $\bar{\mu}$. The other is to replace $f(\varepsilon)$ by the average distribution function $\bar{f}(\varepsilon)$. The simplest two possibilities, which in fact have been used in literature [8] to discuss various issues connected with transport across the system at hand are the following:

$$
\bar{\mu}=\frac{\mu_{L}+\mu_{R}}{2}
$$

or

$$
\bar{f}(\varepsilon)=\frac{f_{L}(\varepsilon)+f_{R}(\varepsilon)}{2}
$$

where $f_{L / R}=\left(e^{\beta\left(\varepsilon-\mu_{L / R}\right)}+1\right)^{-1}$, is the equilibrium distribution function for the left/right lead. The immediate objection against the above proposals is that both give slightly 
different results, except at equilibrium and none of them is well justified. Equal weights assigned to $\mu_{L(R)}$ or $f_{L(R)}(\varepsilon)$ may possibly be also justified for symmetrically coupled systems only.

Below we shall present an approach which allows the correct calculation of the charge $n$ in non equilibrium systems. To make the presentation of it as simple as possible we shall resort to the specific model describing the quantum dot coupled to two external leads. We use here the Anderson-Hubbard model [9]

$$
H=\sum_{\lambda k \sigma}\left(\varepsilon_{\lambda k}-\mu_{\lambda}\right) c_{\lambda k \sigma}^{+} c_{\lambda k \sigma}+E_{d} \sum_{\sigma} d_{\sigma}^{+} d_{\sigma}+U n_{\uparrow} n_{\downarrow}+\sum_{\lambda k \sigma}\left(V_{\lambda k} c_{\lambda k \sigma}^{+} d_{\sigma}+\text { h.c. }\right) .
$$

Here $\lambda=R, L$ denote the right $(R)$ or left $(L)$ lead in the system. The parameters have the following meaning: $c_{\lambda k \sigma}^{+}\left(c_{\lambda k \sigma}\right)$ denote creation (annihilation) operator for a conduction electron with wave vector $\vec{k}$, spin $\sigma$ in the lead $\lambda, V_{\lambda k}$ is the hybridization matrix element between conduction electron of energy $\varepsilon_{\lambda k}$ in a lead $\lambda$ with chemical potential $\mu_{\lambda}$ and localized electron on the dot. $E_{d}$ is the single particle energy of electrons in the dot. $n_{\uparrow}=d_{\uparrow}^{+} d_{\uparrow}$ is the number operator for electrons with spin up localized on the dot and $U$ is the (repulsive) interaction energy between two electrons on the dot.

The correct way to calculate the charge on the dot under non equilibrium conditions is to use non equilibrium Green's function (GF) of Keldysh [10, 8]. In this technique the average charge on dot at time $t$ is given by

$$
\langle n(t)\rangle=\sum_{\sigma}\left\langle d_{\sigma}^{+}(t) d_{\sigma}(t)\right\rangle=-i \sum_{\sigma} G_{\sigma}^{<}(t, t) .
$$

where $G_{\sigma}^{<}(t, t)$ is diagonal (in time indices) element of the Keldysh "lesser" GF

$$
G_{\sigma}^{<}\left(t, t^{\prime}\right)=i\left\langle d_{\sigma}^{+}(t) d_{\sigma}\left(t^{\prime}\right)\right\rangle
$$

This function is the $(1,2)$ matrix element of the following contour ordered Green's function matrix

$$
\widehat{G}\left(t, t^{\prime}\right)=-i\left\langle T_{c} d(\tau) d^{+}\left(\tau^{\prime}\right)\right\rangle=\left(\begin{array}{cc}
G_{c}, & G^{<} \\
G^{>}, & G_{\bar{c}}
\end{array}\right),
$$

where $T_{c}$ is a complex time contour ordering operator. The time contour starts above time axis at $t_{0}=-\infty$ passes through $t$ and $t^{\prime}$ and returns back to $t_{0}=-\infty$ below time axis. $G_{c}=-i\left\langle T d(t) d^{+}\left(t^{\prime}\right)\right\rangle$ is the usual time ordered (causal) GF for both times on the upper branch of the contour, while $G_{\bar{c}}\left(t, t^{\prime}\right)$ is antitime ordered GF with both 
time arguments on the lower branch. Greater $\left(G^{>}\right)$and lesser $\left(G^{<}\right)$GFs are distribution functions: $G^{>}\left(t, t^{\prime}\right)=-i\left\langle d(t) d^{+}\left(t^{\prime}\right)\right\rangle$. Not all this functions are independent.

It turns out that the calculation of the "lesser" GF is easy for noninteracting quantum dot i.e. for $U=0$. It can be found directly in the following way. Because, the Keldysh contour ordered GF, possesses the same perturbation expansion as the corresponding equilibrium GF [10] it is possible to write down the matrix Dyson equation

$$
\widehat{G}=\widehat{G}_{0}+\widehat{G}_{0} \widehat{\Sigma} \widehat{G}
$$

The theorem due to Langreth [11] allows the transition from contour ordered to real axis functions and one gets 8

$$
G^{<}=\left(1+G^{r} \Sigma^{r}\right) G_{0}^{<}\left(1+\Sigma^{a} G^{a}\right)+G^{r} \Sigma^{<} G^{a}
$$

where $G^{r(a)}$ denotes retarded (advanced) GF.

For noninteracting quantum dot $(U=0)$ exact expression for the retarded dot GF reads

$$
G_{\sigma}^{r}(\omega)=G_{0 \sigma}^{r}(\omega)+G_{0 \sigma}^{r}(\omega) \Sigma^{r}(\omega) G_{\sigma}^{r}(\omega)
$$

with

$$
\Sigma^{r}(\omega)=\sum_{\lambda k}\left|V_{\lambda k}\right|^{2} G_{0 \lambda k}^{r}(\omega),
$$

where $G_{0 \lambda k}^{r}(\omega)=\left(\omega-\varepsilon_{\lambda k}+i 0\right)^{-1}$ is the Green's function of the lead $\lambda$. From this one immediately finds

$$
\begin{aligned}
\Sigma^{<}(\omega) & =\sum_{\lambda k} V_{\lambda k}^{2} G_{0 \lambda k}^{<}(\omega)=i \sum_{\lambda k}\left|V_{\lambda k}\right|^{2} \delta\left(\omega-\varepsilon_{\lambda k}\right) f_{\lambda}(\omega)= \\
& =2 \pi i \sum_{\lambda} \Gamma_{\lambda}(\omega) f_{\lambda}(\omega)
\end{aligned}
$$

where we have used symbol $\Gamma_{\lambda}(\omega)$ to denote the average coupling of the dot to lead $\lambda$; $\Gamma_{\lambda}(\omega)=2 \pi \sum_{k}\left|V_{\lambda k}\right|^{2} \delta\left(\omega-\varepsilon_{\lambda k}\right)$. Noting that $G^{<}(t, t)=\int \frac{d \omega}{2 \pi} G^{<}(\omega)$ and using formula (9) we get

$$
\langle n\rangle=\sum_{\sigma} \int d \omega G^{r}(\omega) \Sigma^{<}(\omega) G^{a}(\omega),
$$

which with help of Eqs. (10) and (12) we further rewrite as

$$
n=\sum_{\sigma} \int d \omega \frac{\sum_{\lambda} \Gamma_{\lambda}(\omega) f_{\lambda}(\omega)}{\sum_{\lambda} \Gamma_{\lambda}(\omega)}\left(-\frac{1}{\pi}\right) \operatorname{Im} G_{\sigma}^{r}(\omega) .
$$


Note that for symmetric coupling $\Gamma_{L}(\omega)=\Gamma_{R}(\omega)$ this formula reduces to the form (四) with average distribution function $\bar{f}(\varepsilon)$ as given by (3). Such an expression for dot occupation has been used in [12] for an interacting dot. We stress, however, that it is valid for noninteracting and symmetrically coupled dot only.

As one can see from the above the main difficulty in calculation of the charge on the dot is connected with calculation of the "lesser" self energy $\Sigma^{<}$. In the noninteracting case the knowledge of the exact expression for self energy (11) allowed easy calculation of exact $\Sigma^{<}$and exact expression for the charge. For interacting dot there is no way to get self energy exactly and one has to approximate it appropriately. One such approximation has been introduced by $\mathrm{Ng}$ [13] and will be discussed latter on. Here we propose to use recently derived equation of motion method [14] and calculate $G^{<}(\omega)$ with the desired accuracy and with approximations consistent with those made in calculation of the current or other response functions of the system.

To make the algebra easy we consider $U=\infty$ limit in the Hamiltonian (雨) and use slave boson technique to handle it. We thus rewrite (4) with help of slave boson operators $b, b^{+}$and use the commutation rules of LeGuillou and Ragoucy [15] to evaluate the necessary quantum brackets. These rules treat exactly the local constraint [16] which for $U=\infty$ prevents double occupancy of the dot. The procedure is simple. One rewrites the Hamiltonian in the form

$$
H^{S B}=\sum_{\lambda k \sigma}\left(\varepsilon_{\lambda k}-\mu_{\lambda}\right) c_{\lambda k \sigma}^{+} c_{\lambda k \sigma}+\varepsilon_{d} \sum_{\sigma} f_{\sigma}^{+} f_{\sigma}+\sum_{\lambda k \sigma} V_{\lambda k}\left(c_{\lambda k \sigma}^{+} b^{+} f_{\sigma}+f_{\sigma}^{+} b c_{\lambda k \sigma}\right)
$$

and calculates the on-dot Green's function $D_{\sigma}^{<}(\omega)=\left\langle\left\langle b^{+} f_{\sigma} \mid f_{\sigma}^{+} b\right\rangle\right\rangle_{\omega}^{<}$using equation (c.f. equation (28b) of [8])

$$
\langle\langle A \mid B\rangle\rangle=g^{<}(\omega)\left\langle[A, B]_{ \pm}\right\rangle+g^{r}(\omega)\left\langle\left\langle\left[A, H_{I}\right] \mid B\right\rangle\right\rangle_{\omega}^{<}+g^{<}(\omega)\left\langle\left\langle\left[A, H_{I}\right] \mid B\right\rangle\right\rangle_{\omega}^{a},
$$

with the third term of $H^{S B}$ taken as a interaction part $H_{I}$ and lower case GFs being the corresponding GFs of the free Hamiltonian $H_{0}$ (consisting of first and second terms of $H^{S B}$ ). The higher order GFs appearing at this stage have been calculated in similar way. In the process we have used the same kind of factorization which one uses calculating the on-dot retarded GF necessary to get Kondo effect. Explicitly we neglected the GFs like $<<c_{\lambda k-\sigma} c_{\lambda^{\prime} k^{\prime} \sigma} f_{-\sigma}^{+} b \mid f_{\sigma}^{+} b>^{a}$ as they describe higher order spin correlations in the leads 
and performed the decoupling

$$
<<c_{\lambda^{\prime} k^{\prime}-\sigma}^{+} c_{\lambda k-\sigma} b^{+} f_{\sigma}\left|f_{\sigma}^{+} b>>^{a} \approx f\left(\varepsilon_{\lambda k}\right)<<b^{+} f_{\sigma}\right| f_{\sigma}^{+} b>>^{a} \delta_{k k^{\prime}} \delta_{\lambda \lambda^{\prime}} .
$$

The resulting "lesser" self-energy takes on simple form

$$
\Sigma^{<}(\omega)=-2 \pi i \sum_{\lambda} \Gamma_{\lambda}(\omega)\left(1+f_{\lambda}(\omega)\right) f_{\lambda}(\omega)
$$

This is our final expression for $\Sigma^{<}(\omega)$ to be used in calculations of the charge $n$.

Now let us rederive the expression for $\Sigma^{<}(\omega)$ using the earlier mentioned approximation introduced by $\mathrm{Ng}$ [13] and often used in the literature [17]. This author proposes to assume that $\Sigma^{<}(\omega)=A \Sigma_{0}^{<}(\omega)$ and $\Sigma^{>}(\omega)=A \Sigma_{0}^{>}$where $A$ is an unknown function and $\Sigma_{0}^{<}(\omega)$ is noninteracting (and thus known) self-energy. For the present example it is given explicitly by (12). This together with the exact relations: $\Sigma^{>}-\Sigma^{<}=\Sigma^{r}-\Sigma^{a}$ and $\Sigma_{0}^{>}-\Sigma_{0}^{<}=\Sigma_{0}^{r}-\Sigma_{0}^{a}$ leads to

$$
\Sigma_{\mathrm{Ng}}^{<}(\omega)=\frac{\Sigma^{r}-\Sigma^{a}}{\Sigma_{0}^{r}-\Sigma_{0}^{a}} \Sigma_{0}^{<}(\omega)
$$

or in explicit form

$$
\Sigma_{\mathrm{Ng}}^{<}(\omega)=-2 \pi i \frac{\sum_{\lambda} \Gamma_{\lambda}(\omega) f_{\lambda}(\omega)}{\sum_{\lambda} \Gamma_{\lambda}(\omega)} \sum_{\lambda} \Gamma_{\lambda}\left(1+f_{\lambda}(\omega)\right),
$$

which again contains the characteristic average distribution function, which for the symmetric coupling reduces to $\bar{f}(\varepsilon)$. Note that this formula agrees with (18) only in equilibrium situation $\mu_{L}=\mu_{R}=\mu$.

It turns out that our formula, Eq. (18) can be rederived by suitably generalizing the Ng's ansatz. To see this note that noninteracting self energy $\Sigma_{0}^{<}(\omega)$ above can be written as a sum of pieces, each of which is connected with one of the leads i.e. $\Sigma_{0}^{<}(\omega)=\Sigma_{\lambda} \Sigma_{0 \lambda}^{<}(\omega)$. One then expects that due to locality of $U$ term in Hamiltonian the interacting self-energy will also be a sum of contributions from different leads $\Sigma^{<}(\omega)=\sum_{\lambda} \Sigma_{\lambda}^{<}(\omega)$. Generalising the ansatz and writing $\Sigma_{\lambda}^{<}(\omega)=A_{\lambda} \Sigma_{0 \lambda}^{<}$independently for each $\lambda$ one immediately reproduces result (18). This shows that the application of equation of motion technique [12 is a correct way to to derive the "lesser" self energy. This technique, contrary to approximate schemes, allows mutually consistent calculations of both retarded and distribution GFs in Keldysh technique. 


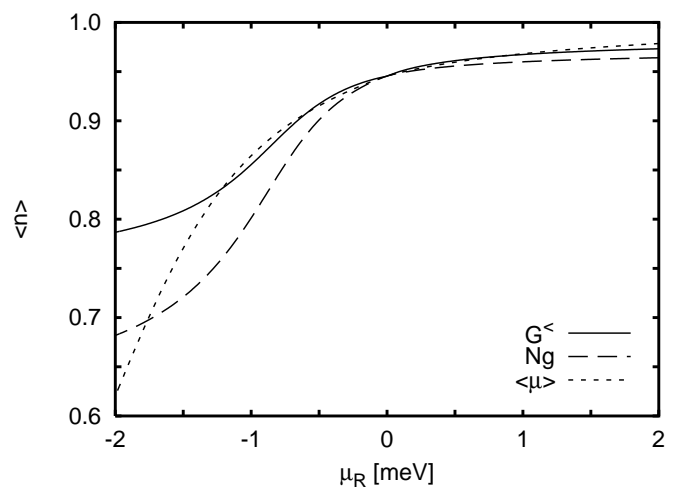

Figure 1: The charge on the quantum dot connected to two external electrodes in dependence of the chemical potential $\mu_{R}$. The parameters are the following: couplings $\Gamma_{L}=\Gamma_{R}=17 \mathrm{meV}$, chemical potential $\mu_{L}=0$ and temperature $T=10^{-4} \mathrm{meV} / k_{B}$.

We have numerically calculated the charge $n$ on the interacting quantum dot (with $U=\infty)$ coupled to leads with different chemical potentials using four formulae discussed in this paper. The results are presented in figures (1) and (2). Figure (1) shows the charge on the dot with $E_{d}=-1 m e V$ as a function of the position of right lead chemical potential $\mu_{R}$ with $\mu_{L}=0$ and for symmetric couplings $\Gamma_{L}(\omega)=\Gamma_{R}(\omega)=\Gamma=17 \mathrm{meV}$. Note tha for symmetric coupling the Ng's approximation give the same results as average distribution function $\bar{f}(\varepsilon)$. In more general case of asymmetric coupling these two approaches lead to different results, but we abandoned this complication here. Solid line show results obtained with help of our formula (18), dashed one is calculated with help of (20), while dotted from (1) with $\bar{\mu}$ given by (2). The crossing of the lines for $\mu_{L}=\mu_{R}=0$ corresponds to equilibrium situation. The differences between the curves get larger for increasing voltage $V=\left(\mu_{L}-\mu_{R}\right) / e$ applied to the system. The large (small) differences between various curves for negative (positive) values of $\mu_{R}$ is due to large (small) values of the on-dot density of states in the energy window between $\mu_{L}$ and $\mu_{R}$. Figure $(2)$ shows the charge on the dot calculated for constant difference $\mu_{L}-\mu_{R}=1 \mathrm{meV}$ as a function of the parameter $E_{d}$. For $E_{d}$ much higher or much lower than both chemical potentials the differences are small as expected because the density of states around $\mu_{L}$ and $\mu_{R}$ is small. The largest differences of the calculated charge are found for such values of $E_{d}$ for which the density of states of electrons on the dot possesses the Kondo resonance.

We conclude by stressing that the calculation of charge on the dot is a necessary step 


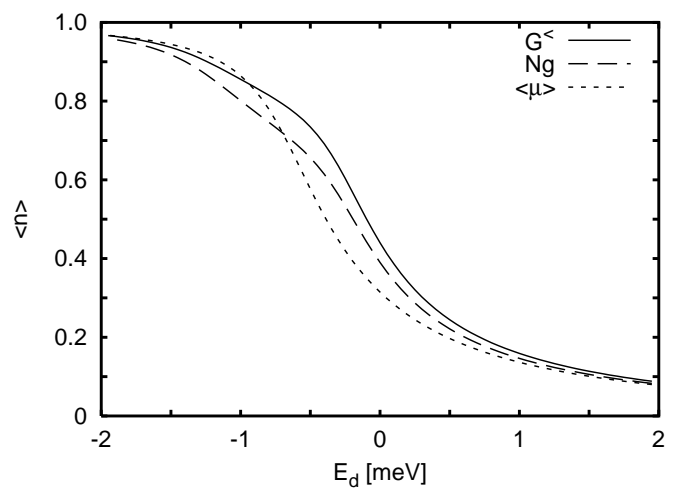

Figure 2: The charge $n$ vs $E_{d}$ for constant difference $\mu_{R}-\mu_{L}=1 \mathrm{meV}$. Other parameters are the same as in Fig.(11).

towards self consistent calculations of the current flowing across quantum dot devices. In some parameter ranges all formulae give only slightly differing results. Generally, however, the differences between values of $n$ calculated by means of different formulae may be as large as 20-30\%. The equation of motion method allows calculation of the lesser self energy consistent with retarded one. This is important as the current flowing in the system is, for proportionate couplings $\Gamma_{L}(\omega)=$ const $\Gamma_{R}(\omega)$, expressed by the retarded Green's function which in turn depends on the charge $n$ on the dot. For general couplings the consistency of approximations is even more important as the current depends on both retarded and lesser GFs and to be consistent one has to treat both self energies on equal footing.

This work has been partially supported by the Committee for Scientific Research under grant 2P03B 10617.

\section{References}

[1] see e. g. "Mesoscopic Quantum Physics" edited by E. Akkermans, G. Montabaux and J. -L. Pickard, North Holland, Amsterdam 1995.

[2] L. P. Kouwenhoven and P. L. McEuen in "Nanotechnology" Ed. G. Timp, Springer Verlag, New York 1999, p. 471.

[3] D. Goldhaber-Gordon, H. Shtrikman, D. Mahalu, D. Abusch-Magder, U. Meirav, M. A. Kastner, Nature 391, 1569 (1998). 
[4] S. M. Cronenwett, S. M. Maurer, S. R. Pate, C. M. Marens, C. I. Durnöz, J. S. Harris, Jr., Phys. Rev. Lett. 81, 5904 (1998); S. M. Cronenwett, T. H. Oosterkamp, and L. P. Kouwenhoven, Science 281, 540 (1998).

[5] T. K. Ng and P. A. Lee, Phys. Rev. 61, 1768 (1989); L. I. Glazman, and M. E. Raikh, Pis'ma Zh. Eksp. Teor. Fiz. 48, 378 (1988) [Engl. transl. JETP] Lett. 47, 452 (1988)]; S. Herschfield, J. H. Davies, and J. W. Wilkins, Phys. Rev. Lett. 67, 3720 (1991).

[6] Y. Meir N. S. Wingreen, and P. A. Lee, Phys. Rev. Lett. 70, 2601 (1993); N. S. Wingreen, and Y. Meir, Phys. Rev. B 49, 110400 (1994).

[7] S. Doniach and E. H. Sondheimer, "Green's functions for Solid State Physicists", Redwood City, CA, Addison Wesley 1982.

[8] H. Haug, A. P. Jauho, "Quantum Kinetics in Transport and Optics of Semiconductors", Springer Verlag, Berlin 1996.

[9] P. W. Anderson, Phys. Rev. 124, 41 (1961).

[10] L. V. Keldysh, Zh. Eksp. Teor. Fiz. 47,1515 (1965) [Sov. Phys. JETP 20, 1018 (1965)].

[11] D. C. Langreth, in "Linear and Nonlinear Electron Transport in Solids", eds. J. T. Devreese and E. van Doren, Plenum Press, New York 1976.

[12] Q. Sun and T. Lin, Physica E 4, 201 (1999).

[13] T. K. Ng, Phys. Rev. Lett. 76, 487 (1996).

[14] C. Niu, D. L. Lin and T. -H. Lin, J. Phys. Cond. Matt. 11, 1511 (1999).

[15] J. C. Le Guillou, E. Ragoucy, Phys. Rev. B 52, 2403 (1995).

[16] M. Krawiec and K. I. Wysokiński, Phys. Rev. B 59, 9500 (1999).

[17] R. Fazio, R. Raimondi, Phys. Rev. Lett. 80, 2913 (1998). 